\title{
„WHEN WILL I BECOME A SCHNITZEL? - I HOPE NEVER. " ECHTE UND FALSCHE FREUNDE DES ÜBERSETZERS IN DER TRANSLATIONSDIDAKTIK
}

\author{
ARTUR DARIUSZ KUBACKI, Univ.-Prof. Dr. habil. \\ Pädagogische Universität Krakau \\ Lehrstuhl für deutsche Sprachwissenschaft \\ am Neuphilologischen Institut \\ ul. Studencka 5, pok. 403, 31-116 Kraków \\ kubart@post.pl
}

URSULA KIERMEIER, M.A.

ursula.kiermeier@gmx.de

\begin{abstract}
Das Phänomen der falschen Freunde des Übersetzers ist sprachenpaarbezogen und beruht darauf, dass ein phonetisch ähnlich wirkendes Wort oder eine analoge Wendung eine oft unterschiedliche Bedeutung besitzt und der Lernende sich durch den Gebrauch eines falschen Freundes öffentlich lächerlich macht, wie im Titel an einem deutsch-englischen Witz veranschaulicht. Insbesondere Politiker sind für ihre falschen Freunde (nicht nur in der Sprache) berüchtigt, früher war es Helmut Kohl, heute der deutsche EU-Kommissar Günther Oettinger. Auch in die Literatur hat das Phänomen Eingang gefunden, wenn wir an die englischen Kommunikationsversuche Kommissar Kluftingers in den Krimis des Allgäuer Autorenduos Klüpfel/Kobr denken. Ein falscher Freund im Translat ist aber nicht nur für Politiker höchstnotpeinlich, sondern gerade auch für Übersetzende und
\end{abstract}




\title{
Artur Dariusz Kubacki: ,, When Will I Become a Schnitzel?...
}

Dolmetschende. In der traditionellen Didaktik haben die Lernenden daher gelernt, die falschen Freunde zu fürchten wie der Teufel das Weihwasser. Es entstehen spezielle Wörterbücher mit falschen Freunden und die Aufgabe der Lernenden ist es, diese verfänglichen Wörter und Wendungen auswendig zu lernen. Das gilt insbesondere für Dolmetscher, denn sie haben anders als Übersetzer in der beruflichen Praxis nicht die Zeit zu überprüfen, ob es sich bei einer sich auf die Lippen drängenden, analogen Formulierung um einen falschen Freund oder einen echten handelt, d.h. um eine im jeweiligen Kontext zulässige und gängige Formulierung in der Zielsprache. Die mit langjähriger Erfahrung als Sprachmittler/In tätigen Autoren möchten am Beispiel des deutsch-polnischen und deutsch-englischen Sprachenpaars auf die Gefahren einer vereinseitigenden Didaktik der falschen Freunde hinweisen, die dazu führen kann, dass Lernende, in der Angst, sich lächerlich zu machen, tatsächliche sprachliche Analogien nicht nutzen. Diese negative Auswirkung der Falsche-Freunde-Didaktik betrifft insbesondere die Fachsprachen Wirtschaft und Recht.

Schlagwörter: faux amis du traducteur, falsche Freunde des Übersetzers, Interferenzfehler, Übersetzungsdidaktik, Dolmetschdidaktik, Fremdsprachendidaktik

\section{„WHEN WILL I BECOME A SCHNITZEL? - I HOPE NEVER.” PRAWDZIWI I FALSZYWI PRZYJACIELE TLUMACZA W DYDAKTYCE TRANSLACJI}

\begin{abstract}
Abstrakt: Zjawisko fałszywych przyjaciół tłumacza odnosi się do pary języków i polega na tym, że słowa lub zwroty o podobnym brzmieniu lub podobnej pisowni mają odmienne znaczenie. Uczący się używający fałszywego przyjaciela mogą ośmieszyć się publicznie, tak jak to zilustrowano w tytule artykułu na przykładzie niemiecko-angielskiego kawału. Szczególnie politycy mają złą sławę, jeśli chodzi o stosowanie fałszywych przyjaciół tłumacza nie tylko w języku, np. kiedyś Helmut Kohl, a dziś niemiecki komisarz UE Günther Oettinger. Zjawisko to pojawiło się również w literaturze: przypomnimy sobie próby komunikacji po angielsku komisarza Kluftingersa w kryminałach duetu Klüpfel/Kobr. Użycie fałszywego przyjaciela w tłumaczeniu przynosi wstyd nie tylko politykom, ale także tłumaczom słowa pisanego i żywego. W tradycyjnej dydaktyce uczący się starają się unikać fałszywych przyjaciół jak diabeł święconej wody. Powstają specjalne słowniki poświęcone wyłącznie fałszywym przyjaciołom tłumacza, zaś zadaniem uczących się jest zapamiętanie takich podchwytliwych słów i zwrotów. Dotyczy to przede wszystkim tłumaczy ustnych, ponieważ inaczej niż tłumacze pisemni w swojej praktyce zawodowej nie mają czasu na sprawdzenie, czy w przypadku cisnącego się na usta, analogicznego sformułowania chodzi o fałszywego czy prawdziwego przyjaciela thumacza, czyli - inaczej mówiąc - czy jest ono dopuszczalne w danym kontekście w języku docelowym. Autorzy niniejszego artykułu, posiadający wieloletnie doświadczenie w roli tłumacza, chcieliby wskazać na przykładzie par języków niemiecki-polski i niemiecki-angielski na niebezpieczeństwa jednostronnej dydaktyki w zakresie fałszywych przyjaciół tłumacza, która może skutkować tym, że uczący się w obawie przed ośmieszeniem nie będą wykorzystywać faktycznych analogii językowych. Ten negatywny wpływ dydaktyki w zakresie fałszywych przyjaciół tłumacza jest szczególnie widoczny w specjalistycznym języku gospodarki i prawa.
\end{abstract}




\title{
Comparative Legilinguistics 29/2017
}

Slowa kluczowe: faux amis du traducteur, fałszywi przyjaciele tłumacza, błędy interferencyjne, dydaktyka tłumaczenia pisemnego, dydaktyka thumaczenia ustnego, dydaktyka języków obcych

\section{„WHEN WILL I BECOME A SCHNITZEL? - I HOPE NEVER.” REAL AND FALSE FRIENDS OF A TRANSLATOR IN TRANSLATION TEACHING}

\begin{abstract}
The phenomenon of false friends of a translator relates to a pair of languages. It occurs when words or phrases that sound or look similar in two languages differ to a significant degree in meaning. Consequently, learners using a false friend make a fool of themselves as it has been illustrated in the title of the article with an English-German joke. Politicians in particular are notorious for using false friends (Helmut Kohl and the German EU commissioner Günther Oettinger might be good examples). This phenomenon appeared in literature as well: for instance attempts at communicating in English by the police officer Kluftingers in crime novels co-written by Klüpfel/Kobr. The use of a false friend shames not only politicians but also translators and interpreters. In traditional foreign language teaching, students are taught to avoid false friends at all costs. There are dictionaries devoted only to false friends and learners are supposed to learn them by heart. Especially interpreters are recommended to do that because, unlike translators, they do not have time while interpreting to check whether a similar word or phrase coming automatically to mind is a false friend or a real one, that is - in other words - whether the word or phrase has the equivalent meaning in the target language. The authors of this article, who have considerable professional experience of translation, would like to point out the dangers of one-sided teaching practice with regard to false friends, taking German and Polish and German and English as examples. Such teaching may discourage learners - for fear of humiliating themselves - from using actual linguistic analogies. This negative impact of the way false friends are taught is particularly visible in the language of economy and law.
\end{abstract}

Keywords: faux amis du traducteur, false friends of a translator, language transfer errors, translation teaching, interpretation teaching, teaching foreign languages

\section{Theoretische Einführung}

Der Termin faux amis du traducteur wurde in die Literatur im Jahre 1928 durch zwei französische Sprachwissenschaftler Maxime Koessler und Jule Derocquiny ${ }^{1}$ eingeführt und ist seit dieser Zeit unter der übersetzten Sprachversion dieses Termins in viele andere Sprachen eingedrungen, z.B. im Deutschen falsche Freunde des

\footnotetext{
${ }^{1}$ M. Koessler, J. Derocquigny. 2008. Les faux amis ou Les trahisons du vocabulaire anglais: Conseils aux traducteurs. Paris.
} 
Artur Dariusz Kubacki: ,, When Will I Become a Schnitzel?...

Übersetzers, im Englischen false friends of a translator, im Polnischen fatszywi przyjaciele tlumacza. Diese bereits sehr bekannte und facettenreiche Erscheinung ${ }^{2}$ weckt bis heute reges Interesse sowohl bei Sprachwissenschaftlern, Glottodidaktikern, Übersetzungsund Dolmetschwissenschaftlern, als auch praktizierenden Dolmetschern und Übersetzern. Wir wollen dagegen dieser Erscheinung unsere Aufmerksamkeit aus übersetzungsdidaktischer Perspektive schenken.

Viele Wissenschaftler bezeichnen das Phänomen der falschen Freunde je nach der bevorzugten Untersuchungsperspektive unterschiedlich. Daher sind sie z.B. in der umfangreichen Literatur auch unter folgenden deutschen Termini bekannt: zwischensprachliche Homonyme, Inter-Homonyme, interlinguale Homonyme, interlinguale Paronyme, lexikalische Scheinidentitäten, scheinbare Äquivalente, Pseudoäquivalente, Pseudointernationalismen, irreführende Fremdwörter, Tautonyme, Fallstricke des Wortschatzes, interlinguale Analogismen (Kuczyński 2003: 259f.), primäre Äquivalentassoziationen (Grosbart 1982: 197ff.).

Sowohl in der deutschen als auch der polnischen Sachliteratur liegen sehr viele Definitionen und Klassifikationen von falschen Freunden vor. Eine ausführliche Besprechung hierzu bietet Ernest Kuczyński (2003) in seinem Beitrag unter dem Titel Ein Diskurs zu geschichtlichen, terminologischen und definitorischen Fragen der ,falschen Freunde des Übersetzers". Nach Meinung von Ernest Kuczyński (2003:279) ${ }^{3}$ werden falsche Freunde definiert als „Lexeme, die (a) sich auf zwei oder mehrere Sprachen beziehen, (b) weitgehend ähnliche bzw. identische lautliche/graphische Form aufweisen, (c) oft gleiche Herkunft haben, (d) in den meisten Fällen Entlehnungen aus einer dritten Sprache sind und (e) aufgrund ihrer in bestimmten Merkmalen übereinstimmenden Identität/Ähnlichkeit (in Bezug auf die Muttersprache) zur falschen, normwidrigen, meist auf der Interferenz beruhenden Verwendung von Wörtern bzw. Wortverbindungen u.a. auf der semantischen Ebene beitragen." Anette Kroschewski (2000: 120) fasst die falschen Freunde noch breiter als

\footnotetext{
${ }^{2}$ Eine umfangreiche bibliografische Zusammenstellung $\mathrm{zu}$ falschen Freunden bietet Ryszard Lipczuk (2000) im publizierten Text unter dem Titel Bibliografia na temat ,faux amis “ sowie auf der Internetseite www.lipczuk.buncic.de (Zugriff: 20.10.2016) in einer zusammen mit Daniel Buncič erarbeiteten online-Bibliographie.

${ }^{3}$ Ähnlich bei Jerzy Pieńkos (2003:187).
} 


\section{Comparative Legilinguistics 29/2017}

Kuczyński auf. Sie zählt zu ihnen nicht nur einzelne Lexeme, sondern auch ganze Strukturen wie z.B. Phraseologismen. Als Beispiel für einen falschen Freund gilt die deutsche Entsprechung ich habe Luft verloren für den polnischen Satz stracitem/-am oddech, anstatt die adäquate Wendung nie moge złapać tchu [ich bin völlig außer Atem/ich bekomme keine Luft mehr] anzuwenden. Darüber hinaus wird bei ihr zwischen strukturellen, morphologischen, stilistischen, pragma- und textlinguistischen falschen Freunden unterschieden.

Przemysław Janikowski und Joanna Krzywda (2011: 115-135) stellen in ihrem Beitrag $\mathrm{zu}$ falschen Freunden und deren Untersuchungssystematisierung eine interessante Geschichte der wissenschaftlichen Recherchen über diese Erscheinung dar. Es stellt sich nämlich heraus, dass die Untersuchungen in drei grundlegende Richtungen gehen: (1) terminologische Ermittlungen, Vereinheitlichung von Definitionen der falschen Freunde sowie (3) Schaffung einer klaren Typologien für sie (Janikowski, Krzywda 2011: 121). Es folgen die Untersuchungen dieses Phänomens, so Janikowski und Krzywda (2011: 122), aus sprachwissenschaftlicher, glottodidaktischer und translatorischer Perspektive, wobei auch die Translationsdidaktik bei diesem Aspekt eine wichtige Rolle zu erfüllen hat.

Die sprachwissenschaftlich ausgerichteten Untersuchungen von falschen Freunden umfassen einerseits Analysen des kontextlosen Wörterbuchwortkorpus, andererseits Analysen der Phraseologie und Kollokationen im Rahmen konfrontativer, auf verschiedene Sprachenpaare fokussierter Arbeiten. Außerdem gibt es auch Beiträge zur Genese und Frequenz von falschen Freunden im Bereich diverser Sprachenpaare sowie konfrontative semantische Analysen verbunden mit graphischen und phonetischen Untersuchungen. Viele Sprachwissenschaftler erstellen ebenfalls Allgemein- und Fachwörterbücher mit falschen Freunden. Das erste Wörterbuch stammt bereits aus dem Jahre 1747 und betrifft Germanismen in der französischen Sprache (de Mauvillon 1747).

Die falschen Freunde stehen auch im Mittelpunkt der Aufmerksamkeit von Glottodidaktikern besonders aus der Perspektive potentieller Fehler, die von den Lernenden begangen werden können. Daher wird diese Erscheinung im Hinblick auf negative Interferenz dargestellt. Untersucht werden hierzu Lehrbücher und selbst Lernende mit ihren authentischen Verhaltensweisen und begangenen Fehlern. 
Artur Dariusz Kubacki: ,, When Will I Become a Schnitzel?...

Schließlich ist auch das Phänomen der falschen Freunde Gegenstand der Untersuchung von Translationswissenschaftlern, und besonders von Translationsdidaktikern. Da werden die sprachwissenschaftlichen und glottodidaktischen Ansätze zusammengeführt. Meistens werden die durch falsche Freunde verursachten Fehler in bereits bestehenden Übersetzungen genauer betrachtet. Auch die Fragen der Pseudo- und falschen Äquivalente in den Fachübersetzungen werden im Hinblick auf die falschen Freunde diskutiert. Janikowski und Krzywda (2011: 127) sind der Auffassung, dass die Untersuchungen zu falschen Freunden zwei „emotionalen Kategorien“" zugeordnet werden können. Die einen Wissenschaftler dämonisieren die Erscheinung der Interferenz und empfehlen noch eine größere Marginalisierung von falschen Freunden, die anderen entmythologisieren dagegen die falschen Freunde. Die neueren Recherchen über diese Erscheinung im Übersetzungs- und Dolmetschprozess sind vor allem auf statistische Untersuchungen gestützt und auf psycholinguistische Erscheinungen wie lexical priming fokussiert.

Wie bereits erwähnt, wurden sehr viele Klassifikationsversuche von falschen Freunden angestellt. Aus Platzgründen präsentieren wir lediglich eine Typologie von einem international anerkannten polnischen Falsche-FreundeWissenschaftler Ryszard Lipczuk, der zu diesem Thema im Sprachenpaar Polnisch-Deutsch Wörterbücher, Übungshefte und viele Beiträge publiziert hat. Lipczuk (2001: 9) klassifiziert faux amis nach einer engen und weiten Auffassung. Bei den ersteren handelt es sich um Wörter zweier oder mehrerer Sprachen, die sich bei gleicher oder ähnlicher (graphischer und/oder phonetischer) Form in ihrem semantischen Gehalt unterscheiden. Solche Lexeme nennt Lipczuk Tautonyme, z.B. dt. Kriminalist [Kriminalbeamter] - pl. kryminalista [Krimineller, Verbrecher]. Zu den faux amis (abgekürzt FF) nach der weiten Auffassung zählt Lipczuk (1992: 139; 2000: 14; 2001: 9):

1) Wörter mit gleicher Bedeutung aber unterschiedlicher Schreibweise (orthographische FF), z.B. dt. Aggression - pol. agresja,

2) Wörter mit bestimmten Unterschieden in der Aussprache (phonetische FF), z.B. dt. Typ [ty:p] - poln. typ [tip $]^{4}$,

\footnotetext{
${ }^{4}$ Der polnische Skispringer Maciej Kot hat einen sprechenden Namen: kot bedeutet Katze. Durch eine falsche Aussprache im Deutschen wurde aus der Katze wider
} 


\section{Comparative Legilinguistics 29/2017}

3) Wörter mit bestimmten grammatischen Unterschieden, z.B. Genus bei Substantiven: dt. das Referat - pol. $\underline{(t e n=d e r)}$ referat,

4) Wörter mit bestimmten Unterschieden in der Wortbildungsstruktur, z.B. dt. absurd - pol. absurdalny,

5) Wörter mit formal unterschiedlicher Form, die zwar global als semantische Entsprechungen gelten, was aber nicht immer zutrifft, z.B. dt. gro $\beta$ - pol. duży [im Deutschen starke Bewölkung und im Polnischen große Bewölkung),

6) Wörter mit gleicher Wortbildungsstruktur, aber unterschiedlicher phonologisch-graphischer Gestalt und unterschiedlicher Bedeutung, z.B. dt. überhören [etwas hören, aber darauf nicht reagieren) - eng. to overhear [etwas zufälligerweise zu hören bekommen],

7) Phraseologismen mit ähnlicher Struktur, aber unterschiedlichen Bedeutungen, z.B. dt. den Kopf verlieren [verwirrt sein] (nur in der Bedeutung 1) - pol. tracić głowe [1. verwirrt sein; 2. dem Charme einer Person erliegen],

8) Wörter einer Sprache, die in einer früheren Zeit eine andere Bedeutung hatten als in der Gegenwartssprache (diachronische FF), z.B. dt. List [früher: Wissen, Fähigkeit, heute: Schlauheit], pol. gładki [früher: schön, heute: glatt, eben),

9) bestimmte Äußerungen mit gleicher (ähnlicher) Form, aber verschiedenen kommunikativen Funktionen (pragmatische FF), z.B. dt. Tschüs! (verwendbar beim Abschied) - pol. Cześć! (verwendbar als Begrüßung und beim Abschied),

10) Einzelwörter (keine Wortpaare), die in einer Sprache gebräuchlich sind, in der anderen aber nicht existieren, z.B. norw. sendemann $=\mathrm{dt}$. Botschafter, Gesandter.

Ryszard Lipczuk (1985: 69) vertritt die Meinung, dass bei Ermittlung der faux amis nach der weiten Auffassung auch solche Faktoren mit zu berücksichtigen sind, wie Stilschicht (z.B. dt. Kaprize $=$ gehobene Schicht - pol. kaprys $=$ neutrale Schicht $)$, territorialer Gebrauch (z.B. Sprachvarietät in Österreich lizitieren [versteigern] - pol. licytować, in der Schweiz: manifestieren [an einer Massenkundgebung teilnehmen] - pol. manifestować), zeitliche 
Zuordnung beruhend auf Unterschieden in der Aktualität des Gebrauchs (z.B. dt. proponieren/vorschlagen - pol. proponować) wie auch Gebrauchshäufigkeit (z.B. dt. Quästion [in der Philosophie: in einer Diskussion entwickelte und gelöste Frage] - pol. kwestia [Frage, Angelegenheit]; es handelt sich dabei um Lexeme, die von einem begrenzten Sprecherkreis verwendet werden und auf ein bestimmtes Gebiet der menschlichen Tätigkeit bezogen sind).

Der Einteilung der faux amis und besonders der Tautonyme, also faux amis nach der engen Auffassung von Lipczuk, liegen Arten der semantischen Parallelen oder Differenzen, also die Arten der semantischen Relationen zwischen einzelnen Lexemen im jeweiligen Sprachenpaar zu Grunde (Lipczuk, 1985: 64-65, 2000: 13). Es sind:

1) Relation der Privativität (ein Lexem einer Sprache weist eine oder mehr als eine Bedeutung auf, die in einer anderen Sprache nicht nachweisbar ist), z.B. dt. Akkreditiv [1. Form bzw. Dokument eines bargeldlosen Zahlungsverkehrs; 2. Beglaubigungsschreiben eines diplomatischen Vertreters, das dem Staatsoberhaupt des fremden Landes überreicht wird] pol. akredytywa (nur in der Bedeutung 1); andere Beispiele: dt. akkreditieren - pol. akredytować, dt. Deszendent - pol. descendent, dt. Karenz-pol. karencja,

2) Relation der Inklusion (die Bedeutung eines Lexems in einer Sprache ist umfassender als die Bedeutung eines entsprechenden Lexems in der anderen Sprache), z.B. dt. Klient [bei einem Anwalt, einer Beratungsfirma] - pol. klient [ein Interessent/Kunde in einem Büro, auf einer Bank, in einem Geschäft, bei einem Anwalt etc.]; andere Beispiele: dt. Fracht - pol. fracht, dt. Aktionär - pol. akcjonariusz, dt. Dividende - pol. dywidenda,

3) Relation der Exklusion (die Bedeutungen der Lexeme schließen sich aus, weil sie keine Gemeinsamkeiten aufweisen), z.B. dt. Reservation (1. Reservat; 2. Sonderrecht; 3. nur Schweiz: Reservierung) - pol. rezerwacja (Buchung, Reservierung von etwas), andere Beispiele: dt. Proband - pol. probant, dt. Etat - pol. etat, dt. Valorisation - pol. waloryzacja,

4) Relation der Kontrarität (die Bedeutungen sind in den beiden Sprachen als gegensätzlich zu betrachten), z.B. dt. Kriminalist [Kriminalbeamter] $\quad$ - pol. kryminalista [Verbrecher]; andere Beispiele: dt. Handicap [Nachteil, 
schlechtere Bedingungen] - pol. handicap [Vorteil, bessere Bedingungen],

5) Relation der Äquipollenz (die Bedeutungen von zwei Lexemen decken sich teilweise, wobei das eine bestimmte Gebrauchsregeln in der einen Sprache aufweist, die das andere entbehrt und umgekehrt), z.B. dt. Intendant [künstlerischer und geschäftlicher Leiter eines Theaters, einer Rundfunkoder Fernsehanstalt] - pol. intendent [geschäftlicher Leiter einer Institution]; andere Beispiele: dt. Kombattant - pol. kombatant, dt. Präsident - pol. prezydent.

Falsche Freunde stellen sowohl in der Übersetzungspraxis als auch in der Fremdsprachendidaktik eine gefährliche Fehlerquelle dar. Übersetzer/Dolmetscher, ob Anfänger oder Fortgeschrittene, sind oft geneigt, ein Fremdwort in einer Sprache als analoges Fremdwort in der anderen Sprache zu verwenden, ohne zu wissen, dass es keine echten Entsprechungen sind. So kommt es oft zu Missverständnissen beim Übertragen aus der einen Sprache in die andere, weil ähnlich aussehende und/oder lautende Wörter andere Bedeutungen aufweisen bzw. unterschiedliche Strukturen darstellen. Aus diesem Grunde ist ihnen besondere Aufmerksamkeit zu schenken.

\section{Beispiele für faux amis im Bereich der Fachsprachen aus translatorischer Praxis}

In der Literatur sind viele Beispiele der faux amis aus der Fachsprache im Bereich der Küche anhand authentischer Speisekarten und Menüs, die deutschsprachigen Kunden in polnischen Restaurants ausgehändigt werden, anzutreffen. Lipczuk (2001: 41-45) hat sie nach lexikalischer, grammatikalischer und orthographischer Ebene gruppiert:

1. Dziczyzna i inne polskie specjalności / Wildheit [Wild] und andere polnischen Spezialitäten (Lexik),

2. Sandacz $w$ maśle / Zander im [in] Butter (Grammatik),

3. Łosoś wędzony z cytryna / Geräucherter Lachs mit Citrone [Zitrone] (Orthographie). 
Artur Dariusz Kubacki: , When Will I Become a Schnitzel?...

Angeführt werden jetzt einige Beispiele der faux amis aus dem Bereich Jura und Wirtschaft ${ }^{5}$ anhand authentischer Übersetzungen und Verdolmetschungen der Kandidaten zum vereidigten Dolmetscher und Übersetzer in Polen, die sich Staatsprüfungen ${ }^{6}$ in den Jahren 20102011 unterzogen haben (Kubacki 2012: 212-234). Die Gruppierung der faux amis erfolgt im Hinblick auf die Art translatorischen Handelns separat für das Übersetzen und das Dolmetschen.

\section{A) Übersetzen (in beide Richtungen)}

1. dystrybucja ciepła - Distribution* der Wärme [Verteilung der Wärme/Wärmeverteilung]

2. generalny wykonawca-Generalausführer* [Generalunternehmer]

3. Krajowy Rejestr Sądowy - *Landesgerichtsregister [nationales/staatliches Gerichtsregister]

4. oddalić pozew $w$ pozostatym zakresie - die Klage ist im sonstigen Umfang abzulehnen* [die Klage ist im Übrigen abzuweisen]

5. umowa o zakazie konkurencji - Vertrag über den* Wettbewerbsverbot [Vertrag über das Wettbewerbsverbot]

6. Należało orzec jak w sentencji wyroku - Man sollte wie in der Sentenz des Urteils entscheiden* [Es war spruchgemäß zu entscheiden]

7. sąd wzywajacy/wezwany - anzurufendes/angerufenes* Gericht [ersuchendes/ersuchtes Gericht]

${ }^{5}$ Gegenstand der Übersetzungen waren eine Vorladung, ein Urteil in einer Zivilsache (Unterhaltsgeld), ein Beschluss über die Bewilligung einer Verfahrenshilfe, ein Lagebericht des Vorstands einer polnischen $\mathrm{GmbH}$, ein Protokoll zur ordentlichen Gesellschafterversammlung, ein Leihvertrag sowie Auszüge aus dem deutschen Gesetz zur Ernennung des Einzelschiedsrichters und zum Begriff Mangel. Bei Verdolmetschungen wurden die Techniken der Prima-Vista-Übersetzung ins Polnische und des Konsekutivdolmetschens ins Deutsche geprüft. Es wurden folgende Texte vom Blatt gedolmetscht: Beschlüsse eines Amtsgerichts, Auszüge aus den AGB, Klage, Vernehmung als Beschuldigter, Prüfung des Jahresabschlusses, Text zur Prüfungs- und Offenlegungspflicht einer mittelgroßen GmbH. Konsekutiv gedolmetscht wurden Ehegelöbnis, Protokolle, Pressemeldungen und Ansprachen.

${ }^{6}$ Nur knapp ein Viertel der Kandidaten besteht die Staatsprüfung zum vereidigten Dolmetscher und Übersetzer in Polen. Das Durchschnittsergebnis im schriftlichen Prüfungsteil liegt bei $120-130$ Punkten (60 \% bis $65 \%$ ) der Höchstpunktzahl. Erforderlich sind jedoch $75 \%$, um zum mündlichen Prüfungsteil zu gelangen. Das Durchschnittsergebnis im mündlichen Prüfungsteil ist dagegen etwas schwächer und liegt bei 110-120 Punkten (unter $60 \%$ ). 


\section{Comparative Legilinguistics 29/2017}

8. uzasadnienie (do wyroku) - Begründung (nur verwendbar in Österreich und der Schweiz) [(Entscheidungs-)Gründe in Deutschland]

9. subjektywnelobjektywne* ustawowe znamiona przestępstwa subjektive/objektive Tatbestände [subiektywne/obiektywne ustawowe znamiona przestępstwa]

10. kwota 1400 zlotych - ein Betrag von 1400 zloty* [Zloty]

11. scedować prawa - Rechte zedieren* [Rechte abtreten]

12. trafić $w$ dziesiątke - ins Zehner* treffen [ins Schwarze treffen]

\section{B) Dolmetschen:}

\section{$\left.B^{6}\right)$ konsekutiv ins Deutsche}

13. Rzeczpospolita Polska-Bundesrepublik* Polen [Republik Polen]

14. Rodzeństwo rodzone/przyrodnie - natürliche (geborene)/unnatürliche* Geschwister [voll-/halbbürtige Geschwister]

15. Będę nosić nazwisko mojego męża - Ich werde den Nachnamen meines Ehemannes tragen* [Amtsdeutsch: Ich werde den Familiennamen meines Ehemannes führen]

16. Powinowactwo w linii prostej - Verschwägerung* in erster* Linie [Schwägerschaft in gerader Linie]

17. Zatwierdzenie sprawozdania finansowego - Genehmigung* des Jahresabschlusses [Feststellung des Jahresabschlusses]

18. Opinia biegłego rewidenta - Gutachten* des Wirtschaftsprüfers [Bestätigungsvermerk des Wirtschaftsprüfers]

19. Małżeństwo jest trwałe - Die Ehe ist haltbar* [beständig]

20. To dla mnie mity obowiązek - Es ist für mich eine nette* Pflicht [eine angenehme Pflicht]

21. W założonej dzisiaj rodzinie - in der heute eingerichteten* Familie [in der heute gegründeten Familie]

\section{B ") vom Blatt ins Polnische}

22. Die Ehe ist gescheitert - małżeństwo zakończyto się* (Die Ehe hat geendet) oder nastapil rozkład matżenstwa* (Die Ehe hat sich zersetzt/ist verfault) [nastąpił rozkład pożycia małżeńskiego]

23. Leichte/grobe Fahrlässigkeit - lekka/duża niedbałość* (leichte/große Nachlässigkeit) oder drobne/znaczace zaniedbanie* (geringe/bedeutende Vernachlässigung) [lekkie/rażące niedbalstwo]

24. Der Beklagte wird verurteilt (BGB) - pozwany zostaje skazany* (StGB) (wörtliche Übertragung) [od pozwanego zasądza się]

An diesen zahlreichen Beispielen lässt sich zeigen, dass die faux amis vor allem die semantische Ebene betreffen. Ab und $\mathrm{zu}$ sind auch Belege für deren Auftreten im Bereich der Schreibweise (Beispiele 9 und 10), der Benennungsmotivik (Beispiel 1 und 2), der 
Wortbildungsstruktur (Beispiel 23), der Grammatik (Beispiel 5), der Phraseologie (Beispiel 12, 20, 21), der Fachphraseologie (Beispiel 4, 6 , 22, 24), der Fachlexik $(11,14,16,17,18,19)$ und sogar der Landes- und Kulturkunde des deutschsprachigen Raums (Beispiel 3, 8, 13) feststellbar.

Auch in der Unterrichtspraxis mit LGBS-Lernenden ${ }^{7}$ lassen sich interessante Beispiele für faux amis im Sprachenpaar DeutschEnglisch feststellen:

25. I work on the third floor. / Ich arbeite im dritten Flur*. [Stock(werk), Korridor]

26. I would like to have a calm hotel room. / Ich möchte ein kaumes* Hotelzimmer. [ein ruhiges Hotelzimmer]

27. That shows / Das schaut* [Das zeigt], wie groß der Bedarf ist.

28. That proves / Das prüft* [Das zeugt davon], wie wichtig das ist.

29. Can you change me* / Können Sie mir Geld wechseln.

30. Could someone loan* a pencil to me? / Kann mir jemand einen Bleistift lehnen? [leihen] (Bei diesem Beispiel ist der Einfluss des falschen Englisch aus der Finanzbuchhaltung zu sehen).

Alle oben präsentierten Beispiele bestätigen, dass es infolge der falschen Freunde bei der Übersetzung zur Entstellung des Inhalts und zur Verletzung der deutschen Sprachregeln durch unkorrekte Wortverbindungen und Fehler im Wortgebrauch bzw. in der Rechtschreibung gekommen ist. Die Fehlübersetzungen können eher auf fehlendes Sprachwissen und Nichtbewahrung der erforderlichen Sorgfalt beim translatorischen Handeln als auf Zeitdruck und Stresseinfluss zurückgeführt werden. Die letzteren zwei Faktoren dagegen sind eine Ursache für die Entstehung von Sprachfehlern beim Dolmetschen.

\section{Vorschläge zur Didaktisierung der faux amis für DaF}

Da die falschen Freunde eine so beachtliche Quelle für Fehler beim Übersetzen und Dolmetschen bilden, soll man ihnen in der

\footnotetext{
${ }^{7}$ Es handelt sich nämlich um Lernende der Lufthansa Global Business Services (LGBS) in Krakau, die Deutsch als Drittsprache lernen und Englisch als zweite Fremdsprache nutzen.
} 


\section{Comparative Legilinguistics 29/2017}

Translationsdidaktik viel Zeit widmen (vgl. Kuczyński 2003: 279). In der polnischen Germanistik werden die falschen Freunde - wie oben gezeigt - sehr intensiv untersucht und die Ergebnisse dieser Untersuchungen für die Didaktik genutzt. Der bereits genannte Stettiner Sprachwissenschaftler Ryszard Lipczuk hat zuerst allein (1990) und später mit einem Redaktionsteam deutsch-polnische Tautonyme-Wörterbücher (1995) herausgegeben. Darüber hinaus wurde den Studierenden ein zweiteiliges Lehrbuch der deutschpolnischen „falschen Freunde“ von Lipczuk und seinen Mitarbeitern am Germanistischen Institut der Universität Stettin zur Verfügung gestellt (1997). Das Lehrbuch besteht aus drei Teilen. Im ersten Teil werden vor allem Übersetzungs- und Ergänzungsübungen angeboten, die je nach Bereich eingeteilt sind, z.B. Sport, Schulwesen, Recht, Wirtschaft/Handel. Im zweiten Teil gibt es deutsche und polnische (präparierte) Texte, die faux amis enthalten, und meist Übersetzungsübungen zum jeweiligen Text. Anschließend wurde im dritten Teil ein kurzes zweisprachiges Glossar erstellt, in dem ausschließlich deutsche und polnische Wörter mit unterschiedlicher Bedeutung verzeichnet sind. Bei deutschen Nomina wurde das grammatische Geschlecht angegeben.

Auch im Sprachenpaar Polnisch-Englisch bestehen einige Lernhilfen, die für die Erscheinung der falschen Freunde sensibilisieren sollen. Grzegorz Szpila hat das englisch-polnische Wörterbuch der falschen Freunde An English-Polish Dictionary of False Friends (2003) sowie dazu das Übungsbuch Make Friends with False Friends. Practice Book (2005) publiziert. Die Übungen sind abwechslungsreich und motivieren die Lernenden zur Arbeit an dieser Erscheinung. Am Ende des Buches steht ein Verzeichnis der englischpolnischen falschen Freunde. Der Autor hat auch einen Schlüssel zum Übungsbuch vorbereitet, daher kann es autodidaktisch verwendet werden.

Es ist jedoch zu warnen, die bilingualen Wörterbücher bei der Übersetzung unkritisch anzuwenden, weil sie wegen einer Vielzahl von falschen Freunden nicht immer glaubwürdig sind (vgl. Czyżewska 2007: 88, Kuczyński 2003: 279). Aus den Untersuchungen der falschen Freunde von Lipczuk $(1995,2000)$ geht eindeutig hervor, dass die bilingualen Großwörterbücher im Sprachenpaar DeutschPolnisch, z.B. das Großwörterbuch Deutsch-Polnisch von Ippoldt und Piprek (1983), auch keine sichere Quelle für Übersetzungen sind. Es ist daher ratsam, die angehenden Übersetzer/Innen auf Fallen der faux 
amis in den populären zweisprachigen Wörterbüchern sensibel zu machen. Dies kann beispielsweise dadurch geübt werden, dass ähnliche Wörter herausgefunden, verglichen und semantische Relationen zwischen ihnen festgestellt werden. Als Hilfe dabei können das Wörterbuch zu falschen Freunden von Lipczuk et al. (1995) sowie geeignete Paralleltexte (Iluk/Kubacki 2006, Kubacki 2011) herangezogen werden.

Auch im Sprachenpaar Englisch-Deutsch gibt es Wörterbücher $\mathrm{zu}$ falschen Freunden. Zu erwähnen ist beispielsweise das Wörterbuch von Barnickel (1992), das aus zwei Teilen besteht. Im ersten wurden Paare von sich nur teilweise unterscheidenden Wörtern zusammengestellt. Der zweite Teil enthält dagegen Paare von Wörtern, deren Bedeutung sich vollständig unterscheidet. Auch Differenzen bei englisch-deutschen idiomatischen Wendungen sind stark vertreten.

Mit den falschen Freunden ist es insoweit leicht, wenn wir sie einmal entdecken, so behalten wir sie auch im Kopf und wir werden dadurch sehr aufmerksam. Der Kontrast zwischen der angenommenen und tatsächlichen Bedeutung des jeweiligen Wortes ist meistens so groß, dass sich uns das Wort mühelos im Gedächtnis einprägt. Wenn wir einmal erfahren, dass ein deutscher Kriminalist einen Mitarbeiter der Kriminalpolizei bezeichnet, und nicht einen polnischen Verbrecher (kryminalista), so brauchen wir uns keiner mnemotechnischen Tricks zu bedienen, um es ein für allemal in der Erinnerung zu behalten.

Was ist jedoch mit solchen faux amis zu machen, die wir uns nicht so leicht merken? Nachstehend folgen in Anlehnung an die Hinweise der Fachübersetzer/Innen im Internet einige Ratschläge: ${ }^{8}$

- Manche faux amis haben zwar eine irreführende Schreibweise, aber sie werden auf Polnisch anders ausgesprochen. Z.B. das englische Wort receipt (Kassenzettel), das dem polnischen Wort recepta (Rezept) ähnelt, wird in beiden Sprachen ganz anders ausgesprochen. Daher ist es wichtig, sich gerade auf die Aussprache zu konzentrieren, um sich das Wort zu merken.

8 Vgl. Fatszywi przyjaciele - jak sobie z nimi radzić?, Językowa Oaza, http://jezykowaoaza.pl/2014/11/faszywi-przyjaciele-jak-sobie-z-nimi/ 20.10.2016); Tautonimy - ,fatszywi przyjaciele thumacza”, https://supertlumacz.pl/jak-ognia-strzezmy-sie-tautonimow/ (Zugriff: 20.10.2016). 
- Bei Substantiven kann man Zeichnungen machen, z.B. bei dem deutschen Wort Mappe (teczka) und dem polnischen mapa (Karte). Am besten ist es, diese Gegenstände mit diversen Farbstiften zu zeichnen, in fremder Sprache zu betiteln und an einem sichtbaren Platz aufzuhängen.

- Es ist ratsam, alle problematischen Wörter im Kontext zu lernen und sie aufzulisten (Erstellen eigener Glossare mit falschen Freunden). Man kann musterhafte Sätze mit derartigen Wörtern bilden oder sogar diese durch Visualisierung der Bedeutung auswendig lernen. Eine andere Methode wäre es, nach einem Text zu greifen, in dem faux amis präsent sind, diese bunt zu markieren und daneben die richtigen Bedeutungen einzutragen.

Viele falsche Freunde lassen sich ohne Zweifel vermeiden, wenn man sich dieser Erscheinung bewusst ist und bei ähnlich aussehenden oder klingenden Wörtern besonders wachsam wird. Außerdem scheint die Problematik der echten Freunde des Übersetzers in der Translationsdidaktik unterschätzt zu sein (vgl. Magdalena Lisiecka-Czop, 2007/2008: 280). Die deutsche Wendung mich trifft der Schlag hat einen echten Freund im Polnischen szlag mnie trafia und kann in der Translationsdidaktik als ein sehr gutes Beispiel für einen echten Freund genutzt werden. Gerade die echten Freunde eignen sich besonders gut als Kompensationsstrategie zum Ausgleich linguistischer Defizite und können bei Erlernung der Translationskunst behilflich sein. Es gilt parallel zur negativen Didaktik der falschen Freunde positive Lernstrategien zur Aneignung der echten Freunde zu entwickeln und ein paralleles, kontextualisiertes Lernen echter und falscher Freunde strukturiert ins Lehrgeschehen für künftige Sprachmittler einzuführen.

\section{Literatur}

Barnickel, Klaus-Dieter. 1992. Falsche Freunde. Ein vergleichendes Wörterbuch deutsch-englisch. Heidelberg: Julius Groos.

Chodacka, Mariola, und Heinz-Uwe Schöffler. 2000. Es gibt auch „echte Freunde” in der Fremdsprache. In: Studia Germanica Posnaniensia 26. S. 169-182.

Czyżewska, Marta. 2007. Fałszywi przyjaciele thumacza, czyli o pułapkach w czasie tłumaczenia. Lingua Legis 15. S. 83-89. 
Artur Dariusz Kubacki: ,, When Will I Become a Schnitzel?...

Mauvillon, Eleazar de. 1747. Remarques sur les germanismes: Ouvrage utile aux Allemands, ausx François, et aux Hollandois. Amsterdam: Mortier.

Fałszywi przyjaciele - jak sobie z nimi radzić?, Językowa Oaza, http://jezykowaoaza.pl/2014/11/faszywi-przyjaciele-jak-sobiez-nimi/ (Zugriff: 20.10.2016).

Greule, Albrecht. 1994. Internationalismen - falsche oder echte Freunde? In: Germanistentreffen Deutschland-Polen 1993, Bonn: Tagungsbeiträge. Regensburg. S. 305-312.

Grosbart, Zygmunt. 1982. Problem mylących podobieństw międzyjęzykowych: Próba definicji i poszukiwania terminologiczne. In: Acta Universitatis Lodziensis: Folia Litteraria 9. S. 197-208.

Haschka, Christine. 1989. Zur Entwicklungsgeschichte der „faux amis“-Forschung. In: Lebende Sprachen 4. S. 148-152.

Hejwowski, Krzysztof. 2009. Klasyfikacja błędów thumaczeniowych teoria i praktyka. In: Jakość i ocena thumaczenia, hrsg. Andrzej Kopczyński, Magdalena Kizeweter, S. 141-161. Warszawa: Wydawnictwo SWPS Academica.

Iluk, Jan, und Artur Dariusz Kubacki. 2006. Wybór polskich i niemieckich dokumentów do ćwiczeń translacyjnych. Warszawa: Promocja XXI.

Janikowski, Przemysław, und Joanna Krzywda. 2011. Fałszywi przyjaciele od lat (Próba systematyzacji badań). In: Historyczne oblicza przekładu, hrsg. Piotr Fast, Anna Car, Wacław M. Osadnik, S. 115-135. Katowice: Śląsk.

Karczewska, Dorota. 2002. O błędach w tłumaczeniu. In: Język rodzimy a język obcy. Komunikacja, przekład, dydaktyka, hrsg. Andrzej Kopczyński, Urszula Zaliwska-Okrutna, S. 129-135. Warszawa: Wydawnictwo Uniwersytetu Warszawskiego.

Kątny, Andrzej. 1993. Falsche Freunde in den deutsch-polnischen Beziehungen. In: Beiträge zur Sprachwissenschaft, Sozio- und Psycholinguistik. Probleme des Deutschen als Mutter-, Fremd- und Zweitsprache, hrsg. Andrzej Kątny, S. 55-66. Rzeszów:

Koessler, Maxime, und Jules Derocquigny. 1928. Les faux amis ou Les trahisons du vocabulaire anglais: Conseils aux traducteurs. Paris: Vuibert. 
Kozłowska, Zofia. 2002. O błędach językowych w tekstach polskich przekładów. In: Język rodzimy a język obcy. Komunikacja, przekład, dydaktyka, hrsg. Andrzej Kopczyński, Urszula Zaliwska-Okrutna, S. 137-147. Warszawa: Wydawnictwo Uniwersytetu Warszawskiego.

Kroschewski, Annette. 2000. False friends und true friends. Ein Beitrag zur Klassifikation des Phänomens der intersprachlichheterogenen Referenz und $\mathrm{zu}$ deren fremdsprachendidaktischen Implikationen. Frankfurt am Main: Peter Lang.

Krzeszowski, Tomasz Paweł. 2000. Fałszywi przyjaciele, konie trojańskie i tymczasowi imigranci. In: Problemy komunikacji międzykulturowej. Lingwistyka. Translatoryka. Glottodydaktyka, red. Barbara Kielar et al., S. 254-264. Warszawa: Graf-Punkt.

Kubacki, Artur Dariusz. 2011. Neue Auswahl deutschsprachiger Dokumente. Warszawa: Translegis.

Kubacki, Artur Dariusz. 2012. Tłumaczenie poświadczone. Status, kształcenie, warsztat tłumacza przysięgłego. Warszawa: Wolters Kluwer Polska.

Kuczyński, Ernest. 2003. Ein Diskurs zu geschichtlichen, terminologischen und definitorischen Fragen der „falschen Freunde des Übersetzers“. Orbis Linguarum 24. S. 255-280.

Kuczyński, Ernest. 2005. Interferenzträchtige Stolpersteine bei der Übersetzung. Das Wesen und die Problematik ,der falschen Freunde des Übersetzers". Włocławek: Wydawnictwo PWSZ.

Lipczuk, Ryszard. 1985. Semantische Relationen im Bereich der lexikalischen Tautonyme im Deutschen und Polnischen. In: Studien zum polnisch-deutschen Sprachvergleich 2. S. 61-72.

Lipczuk, Ryszard. 1989. Zum Problem der „falschen Freunde” des Übersetzers. In: Theorie und Praxis der deutsch-polnischen Konfrontation und Translation, red. Andrzej Kątny, S. 41-52. Rzeszów: Wydawnictwo Wyższej Szkoły Pedagogicznej.

Lipczuk, Ryszard. 1991. Falsche Freunde des Übersetzers. Forschungsprobleme und Streitfragen. In: Akten des VIII. Internationalen Germanisten-Kongresses Tokyo 4, hrsg. Yoshinori Shichiji, S. 404-411. München: Mosaik Verlag.

Lipczuk, Ryszard. 1992. Internacjonalizmy a „fałszywi przyjaciele thumacza". In: Język a kultura 7, hrsg. Jolanta Maćkiewicz, 
Artur Dariusz Kubacki: ,, When Will I Become a Schnitzel?...

Janusz Siatkowski. S. 135-143. Wrocław: Wydawnictwo Centralnego Programu Badań Podstawowych.

Lipczuk, Ryszard et al. 1995. Niemiecko-polski słownik tautonimów. Warszawa: PWN.

Lipczuk, Ryszard et al. 1997. Von Artisten, Illusionisten, Kriminalisten und anderen falschen Freunden. Übungen und Texte für das Sprachenpaar Polnisch-Deutsch. Teil I: Buchstaben A-K. Szczecin: Wydawnictwo Naukowe Uniwersytetu Szczecińskiego.

Lipczuk, Ryszard et al. 1997. Von Piloten, Pionieren, Potentaten und anderen falschen Freunden. Übungen und Texte für das Sprachenpaar Polnisch-Deutsch. Teil II: Buchstaben L-Z. Szczecin: Wydawnictwo Naukowe Uniwersytetu Szczecińskiego.

Lipczuk, Ryszard. 2000. „Fałszywi przyjaciele tłumacza“ w słownikach niemiecko-polskich. In: Problemy frazeologii i leksykografii, red. Andrzej Kątny, Krzysztof Hejowowski, S. 13-21. Olecko: Wszechnica Mazurska.

Lipczuk, Ryszard. 2000. Bibliografia na temat „faux amis”. In: Problemy frazeologii i leksykografii, hrsg. Andrzej Kątny, Krzysztof Hejowowski, S. 175-200. Olecko: Wszechnica Mazurska.

Lipczuk, Ryszard. 2001. Falsche Freunde des Übersetzers und Tautonyme. In: Lehr- und Übungsbuch zur deutschpolnischen und polnisch-deutschen Übersetzung, hrsg. Ryszard Lipczuk, Erwin Leibfried, Krzysztof Nerlicki, Sascha Feuchert, S. 9-12. Szczecin: Giga.

Lipczuk, Ryszard. 2003. Internacjonalizmy a „fałszywi przyjaciele thumacza“. Orbis Linguarum 24. S. 135-143., http://docslide.pl/documents/19ryszard-lipczukinternacjonalizmy-a-falszywi-przyjaciele-tlumacza.html (Zugriff: 20.10.2016).

Lipczuk, Ryszard, Buncič, Daniel. An online hypertext bibliography on false friens. www.lipczuk.buncic.de (Zugriff: 20.10.2016).

Lisiecka-Czop, Magdalena. 2007/2008. „Prawdziwi przyjaciele thumacza" a dydaktyka i praktyka przekładu. Rocznik Przekładoznawczy 3/4. Studia nad teorią, praktyką i dydaktyka przekładu. S. 271-282.

Pieńkos, Jerzy. 2003. Podstawy przekładoznawstwa. Od teorii do praktyki. Kraków: Kantor Wydawniczy Zakamycze. 
Piprek, Jan, und Juliusz Ippoldt. 1983. Wielki słownik niemieckopolski. Das Großwörterbuch Deutsch-Polnisch. Warszawa: Wiedza Powszechna.

Schatte, Christoph. 1990. Internationalismen und falsche Freunde in den Lexika des Deutschen und Polnischen. In: Internationalismen. Studien zur interlingualen Lexikologie und Lexikographie, hrsg. Peter Braun, Burkhard Schaeder, Johannes Volmert, S. 87-94. Tübingen: Niemeyer.

Szpila, Grzegorz. 2003. An English-Polish Dictionary of False Friends. Angielsko-polski słownik fałszywych przyjaciół. Kraków: Egis.

Szpila, Grzegorz, 2005. Make Friends with False Friends. Practice Book. Kraków: Egis.

Tautonimy „fałszywi przyjaciele thumacza”, https://supertlumacz.pl/jak-ognia-strzezmy-sie-tautonimow/ (Zugriff: 20.10.2016). 
